\title{
Estimación del área basimétrica en masas maduras de Pinus Sylvestris en base a una única medición del escáner láser terrestre $(T L S)$
}

\author{
Estimating basal area in mature stands of Pinus Sylvestris \\ using single-scan terrestrial laser scanner (TLS)
}

Molina-Valero, Juan Alberto'; Ginzo Villamayor, María José; Novo Pérez, Manuel Antonio $^{3}$; Álvarez-González, Juan Gabriel ${ }^{1}$; Pérez-Cruzado, César ${ }^{1 *}$

${ }^{1}$ Unidad de Gestión Ambiental y Forestal Sostenible (UXAFORES), Departamento de Ingeniería Agroforestal, Escuela Politécnica Superior de Ingeniería, Universidade de Santiago de Compostela, Benigno Ledo s/n,

Campus Terra, 27002 Lugo, Spain

juanalberto.molina.valero@usc.es

${ }^{2}$ Departamento de Estadística, Análisis Matemático y Optimización, Universidad de Santiago de Compostela, Facultad de Matemáticas, Rúa Lope Gómez de Marzoa s/n. Campus Vida. Santiago de Compostela Spain.CP.15782. mariajose.ginzo@usc.es

${ }^{3}$ Instituto Tecnológico de Matemática Industrial (ITMATI), Edificio Instituto Investigaciones Tecnológicas, planta -1. Rúa de Constantino Candeira s/n. Campus Vida. Santiago de Compostela Spain. CP.15782.

manuelantonio.novo.perez@usc.es 


\title{
Resumen
}

El láser escáner terrestre (TLS) ha surgido como un nuevo dispositivo de tecnología LiDAR con un gran potencial para ser implementado en inventarios forestales (IF). En este trabajo se ha desarrollado un algoritmo con el que se obtienen métricas capaces de estimar el área basimétrica a nivel de parcela $(G)$ en base a una única medición del TLS. El estudio se ha realizado en masas maduras de Pinus sylvestris que han sido inventariadas mediante una red de 40 parcelas que cubren casi por completo el área de distribución y rango de calidades de la especie en España. Este algoritmo se compone de cuatro pasos fundamentales: (1) normalización de la nube de puntos obtenida con el TLS, (2) identificación de los árboles, (3) reducción de la densidad de la nube de puntos, y (4) obtención de las métricas relacionadas con el G. Los mejores resultados se han obtenido con el $G$ estimada en parcelas de $7 \mathrm{~m}$ de radio, alcanzando un coeficiente de correlación de Pearson de 0.86 . Esto ha permitido obtener modelos de regresión lineal simple con valores de $R^{2}$ adj y $R E C M$ de 0.75 y $10.66 \mathrm{~m}^{2}$ para toda la colección de parcelas, y 0.82 y $8.57 \mathrm{~m}^{2}$ para las parcelas agregadas por sitio. Aunque estos resultados sugieren que el TLS podría ser útil para la estimación del $G$ en pinares de $P$. sylvestris, sería necesario contrastarlo en masas que cubran todos los estados de desarrollo para validar su uso en un mayor rango de estados estructurales.

Palabras clave: Inventario forestal, LiDAR, Métricas, Teledetección, Variables dasométricas.

\begin{abstract}
Terrestrial Laser Scanning (TLS) with LiDAR devices has emerged as a new technique of high potential value for implementation in forest inventories (FI). In this study we developed an algorithm to produce stand basal area metrics $(G)$. The research was performed in 40 plots established in mature Pinus sylvestris stands covering the area of distribution and range of site qualities for this species in Spain. The proposed algorithm was obtained in four main steps: (1) normalization of point clouds obtained by TLS; (2) identification of individual trees; (3) reduction of the point cloud density; and (4) determination of $G$ metrics. The $G$ estimated in plots of $7 \mathrm{~m}$ of radius yielded the best results, with a Pearson correlation coefficient value of 0.86 . This enabled us to produce a linear regression model with values of 0.75 for $R^{2}$ adj and 10.66 $\mathrm{m}^{2}$ for RMSE across all plots. Examination of the linear regression model by site yielded higher values of $R^{2} a d j$ and RMSE, of respectively 0.82 and $8.57 \mathrm{~m}^{2}$. Although the results indicate that TLS is a good tool for estimating $G$ in mature $P$. sylvestris stands, further research covering all stages of development is required for comparison of $G$ values estimated in stands with different structures.
\end{abstract}

Keywords: Forest inventory, LiDAR, Metrics, Remote sensing, Dasometric variables. 


\section{Introducción}

La estimación de los recursos forestales es de vital importancia para la gestión y planificación de los servicios ecosistémicos a cualquier escala. Estas estimaciones son llevadas a cabo principalmente mediante técnicas de inventario forestal (IF), las cuales han experimentado una revolución en las últimas décadas debido a la introducción de nuevos dispositivos de medición forestal. De todos los dispositivos de medición remota basados en tecnología láser cabe destacar el láser escáner terrestre (TLS) como una potencial fuente de información para su uso en IF, debido entro otras ventajas, a su capacidad de realizar mediciones de forma inequívoca, objetiva y reproducible (Van Leeuwen y Nieuwenhuis, 2010), siendo el dispositivo láser que ofrece mejor calidad en la obtención de nubes de puntos (Liang et al., 2018). Esto ha provocado un enorme interés por la implementación del TLS en la obtención de variables forestales, y prueba de ello es el ambicioso proyecto internacional "TLS benchmarking Project" (Liang et al., 2018), que recoge los principales logros alcanzados con este dispositivo en la investigación forestal durante los últimos 20 años.

A pesar de las ventajas que reúne el TLS, aún no se ha consolidado como una herramienta operativa en IF por varios motivos, aunque se considera que la automatización en el procesado de los datos mediante un software fácilmente accesible es el principal factor limitante (Liang et al., 2016). Otros factores que limitan su aplicación práctica y que cabría mencionar son el tiempo de adquisición de los datos, que podría verse reducido con un único escaneo, y el coste del propio equipo. Pero para lograr esta consolidación también sería importante que estas nuevas técnicas se focalicen en la obtención de atributos importantes para la gestión forestal. Entre estos atributos destaca el área basimétrica $(G)$ como una de las variables dasométricas más importantes para la gestión forestal (Clutter et al., 1983, p. 71). En este sentido, hay estudios en los que $G$ ha sido estimada en base a una única medición de TLS implementando técnicas de muestreo relascópico (Strahler et al., 2008; Lovell et al., 2011), mediante la agregación de las áreas basimétricas de los árboles identificados (Yao et al., 2011, Moskal y Zheng, 2012), e incluso para bosques hipotéticos generados con simulaciones de Monte Carlo (Corona et al., 2019). Sin embargo, no se encuentran trabajos recientes de esta índole con datos reales. También cabe destacar otros trabajos donde se ha estudiado la estimación del $G$ mediante LiDAR aéreo (Gobakken y Næsset, 2004; Holmgren, 2004), incluso mediante el uso de datos de baja resolución del PNOA (Guerra-Hernández et al., 2016; Arias-Rodil et al., 2018). La tecnología LiDAR también se ha incorporado a vehículos aéreos no tripulados, encontrando trabajos como el de Brede et al. (2017) donde se compara la estimación del diámetro normal $(d)$ entre este dispositivo y el TLS.

Aunque son muchos los algoritmos desarrollados para procesar los datos del TLS (Hopkinson et al., 2004; Moskal y Zheng, 2012; Olofsson et al., 2014; Cabo et al., 2018), algunos de estos tienen un alto coste computacional que se ve aumentado cuando se trata de escaneos múltiples, los cuales crean voluminosas nubes de 
puntos que deben ser corregistradas (Lovell et al., 2011). No obstante, estudios recientes han demostrado que se pueden obtener buenas correlaciones entre las variables de interés y sus estimaciones con el TLS reduciendo la densidad de la nube de puntos (Torralba et al., 2019), lo cual podría mejorar la eficiencia del procesado de los datos. Otro inconveniente que presentan estos algoritmos, especialmente para la estimación de variables de rodal son las oclusiones, las cuales son una limitación importante cuando se trabaja con un único escaneo (Newnham et al., 2015). Para tratar de reducir su efecto sobre las estimaciones se han planteado dos líneas de actuación:

1. Implementar correcciones basadas en principios geométricos para los árboles no detectados (Ducey y Astrup, 2013).

2. Trabajar solo con los árboles detectados, metodología que ha sido menos discutida en la literatura (Liang et al., 2016).

El objetivo de este trabajo es la estimación del $G$ en base a un único escaneo de TLS en masas maduras de Pinus sylvestris en España, para lo que se emplea una red de 40 parcelas que cubren casi la totalidad del área de distribución y de las condiciones de calidad de estación observadas para la especie en España. Para ello se ha desarrollado un algoritmo capaz de procesar las nubes de puntos obtenidas con el TLS, obteniendo como resultado una serie de métricas correlacionadas con el $G$ que se han utilizado para ajustar modelos lineales con los que se puede estimar el $G$ en parcelas circulares de pequeñas dimensiones.

\section{Material y métodos}

\subsection{Adquisición de los datos}

Los datos de campo fueron adquiridos por la Unidad de Gestión Ambiental y Forestal Sostenible (UXAFORES) de la Universidad de Santiago de Compostela (USC). La información proviene de una red de 40 parcelas pertenecientes a masas maduras de $P$. sylvestris con al menos un $90 \%$ de dominancia en $G$, localizadas a lo largo del área de distribución de esta especie en España (Fig. 1). El muestreo ha sido diseñado para cubrir tanto el rango latitudinal como altitudinal de la especie con la finalidad de obtener una muestra que cubra las diferentes calidades de estación de este tipo de masas. Los valores de las principales variables dasométricas se pueden ver en la Tabla 1 .

Las parcelas de inventario han sido circulares de área fija y se han medido todos los árboles con diámetro superior a $12.5 \mathrm{~cm}$. Para cada árbol se han medido las principales variables dendrométricas, utilizando una cinta diamétrica con una apreciación de $0.1 \mathrm{~cm}$ para el $d$ y un hipsómetro digital Vertex IV HAGLOF con una apreciación de $0.1 \mathrm{~m}$ para la altura total. Además, se han obtenido las coordenadas 


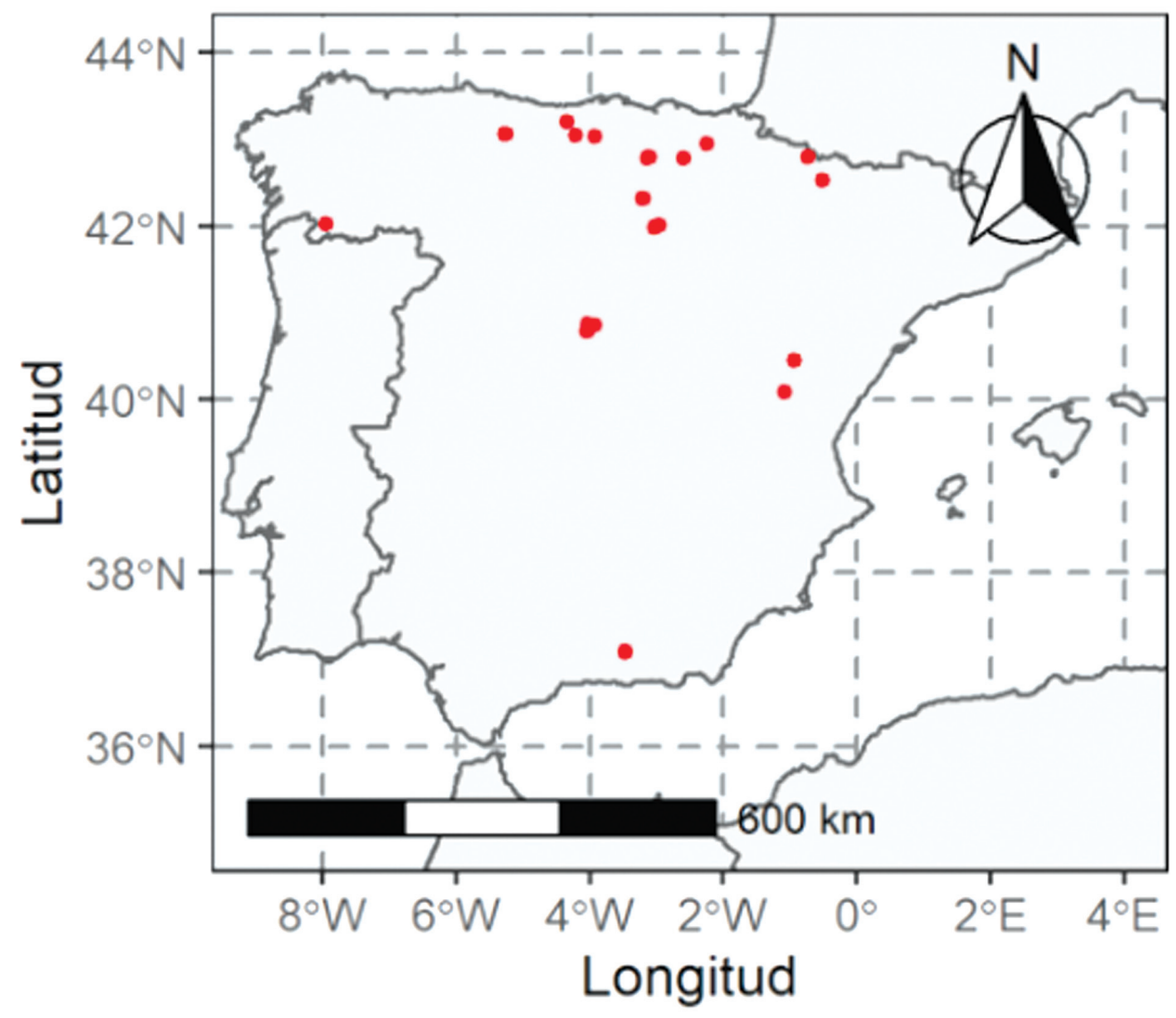

Figura 1. Distribución de las 40 parcelas de inventario en masas maduras de P. sylvestris.

Tabla 1. Principales variables dasométricas de las 40 parcelas de $P$. sylvestris ( $N$ : densidad, $G$ : área basimétrica, $H_{0}$ : altura dominante y $D_{0}$ : diámetro dominante).

\begin{tabular}{lcc}
\hline \multicolumn{1}{c}{ Variable } & Media $($ Min - Max $)$ & SD \\
\hline$N\left(\right.$ pies ha $\left.^{-1}\right)$ & $325.69(45.84-662.08)$ & 135.90 \\
$G\left(\mathrm{~m}^{2} \mathrm{ha}^{-1}\right)$ & $48.82(16.47-74.42)$ & 11.87 \\
$H_{0}(\mathrm{~m})$ & $21.08(10.55-33.55)$ & 5.39 \\
$D_{0}(\mathrm{~cm})$ & $57.55(39.22-82.65)$ & 9.84 \\
\hline
\end{tabular}

polares de cada árbol mediante la medición de los ángulos azimutales y distancias horizontales desde el centro de la parcela, para lo que se ha utilizado el dispositivo TruPulse $360^{\circ} \mathrm{R}$, el cual tiene apreciaciones de $0.1^{\circ}$ y $0.1 \mathrm{~m}$ respectivamente. Para el muestreo LiDAR se ha empleado un TLS FARO Laser Scanner Focus ${ }^{3 \mathrm{D}}$ X 130, con el que se ha realizado un único escaneo desde el centro de la parcela. Las propiedades y parámetros de configuración de adquisición del TLS que han sido definidos en los escaneos pueden verse en la Tabla 2. 
Tabla 2. Propiedades y parámetros definidos del TLS FARO Laser Scanner Focus ${ }^{3 \mathrm{D}}$ X 130 para la adquisición de datos.

Diámetro del haz
Divergencia del haz
Rango horizontal
Rango vertical
Espaciamiento entre puntos
Tamaño del escaneo
Duración del escaneo
Resolución (vertical / horizontal)
Rango

Diámetro del haz

Divergencia del haz

Rango horizontal

$0.011^{\circ}(1 / \mathrm{e})$

$0.0^{\circ}$ hasta $360.0^{\circ}$

$-60.0^{\circ}$ hasta $90.0^{\circ}$

$7.67 \mathrm{~mm} / 10 \mathrm{~m}$

$8248 \times 3414$ (ptos.)

$8.54 \mathrm{~min}$

$0.009^{\circ} / 0.009^{\circ}$

$0.6 \mathrm{~m}-130 \mathrm{~m}$

\subsection{Procesado de los datos}

El algoritmo empleado para procesar los datos se basó en una serie de pasos principales similares a la mayoría de los algoritmos publicados en la literatura (p. ej. Cabo et al., 2018) (Fig. 2), a excepción del point crooping que es una aportación nueva de este trabajo. Los datos crudos adquiridos con el TLS se transformaron a archivos LAS, que es el formato más utilizado con datos LiDAR y con el que funcionan la mayoría de programas informáticos. A partir de este paso, todo el procesado se llevó a cabo con el software estadístico R (R Core Team, 2019).

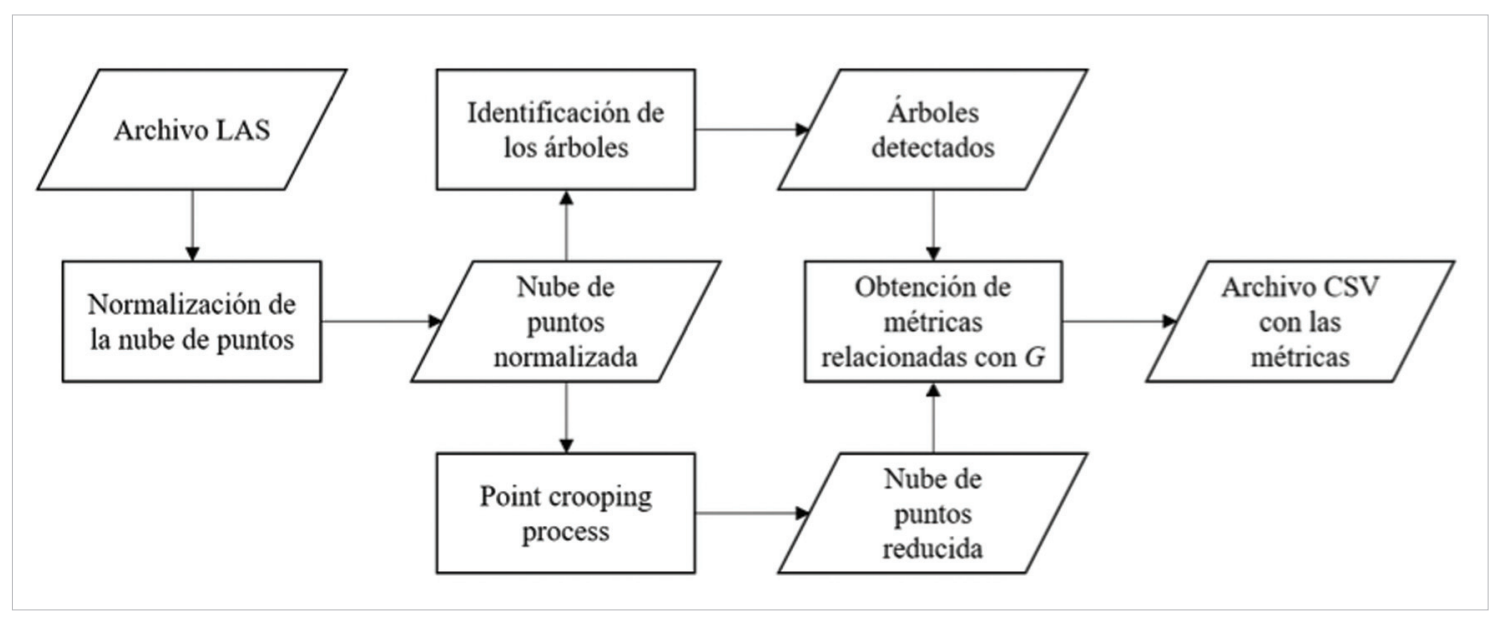

Figura 2. Esquema general del algoritmo implementado.

\subsubsection{Normalización de la nube de puntos}

El proceso de normalización consiste en asignar a los puntos, originalmente con valores de altitud, valores de alturas relativas con respecto al suelo. Para llevar a 
cabo este proceso se han utilizado algunas de las funciones incluidas en el paquete 'lidR' (Roussel et al., 2019). Los pasos realizados han sido:

1. Clasificación de los puntos como suelo o no suelo con la función lasground mediante el algoritmo CSF descrito en Zhang et al. (2016).

2. Generación del modelo digital del terreno (MDT) con los puntos clasificados como suelo mediante el método de interpolación de la distancia inversa ponderada incluido en la función grid-terrain.

3. Normalización de la nube de puntos utilizando el MDT generado y el archivo LAS de la nube de puntos como inputs en la función lasnormalize. Por último, se calcularon las coordenadas cartesianas $(\mathrm{x}, \mathrm{y}, \mathrm{z})$ y esféricas $(r, \theta, \varphi)$ de cada punto.
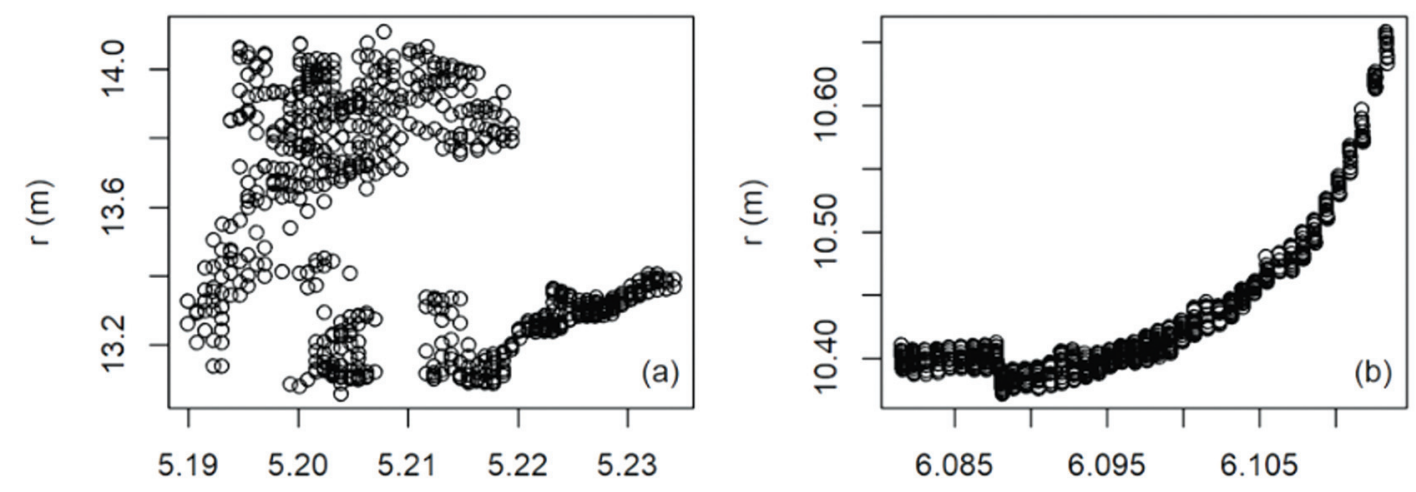

$\mathrm{t}(\mathrm{rad})$
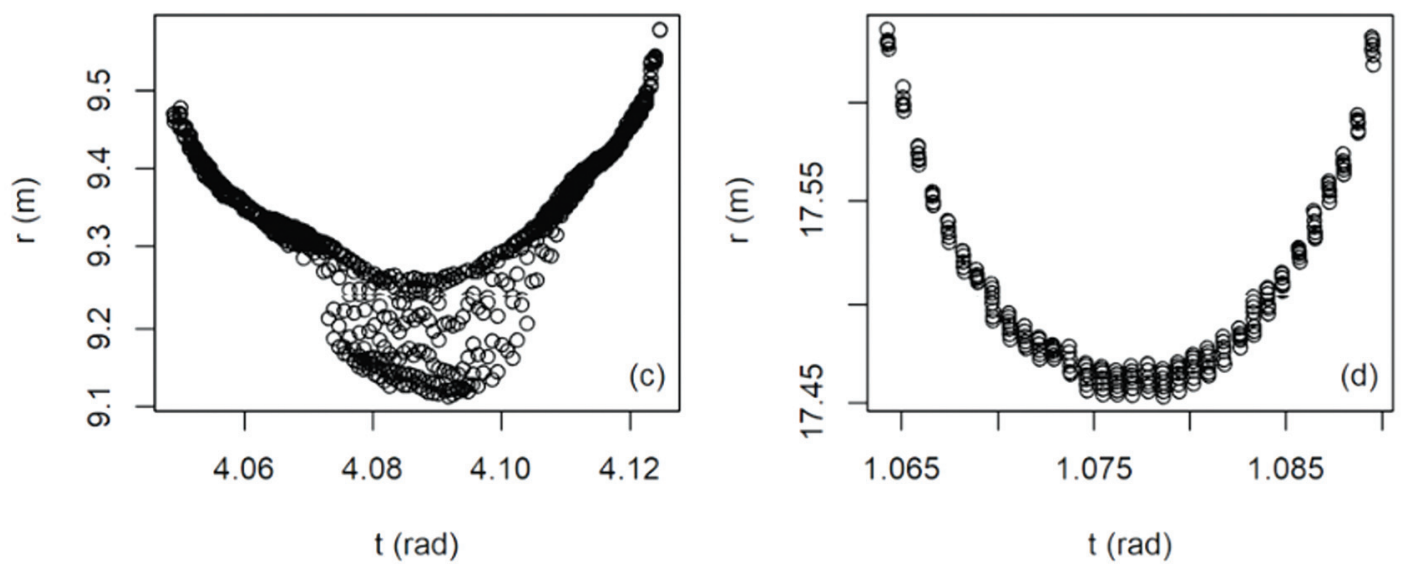

Figura 3. Posibles clústeres obtenidos para las diferentes secciones. Clúster que no pertenece a un árbol (a); árbol con una oclusión parcial (b); árbol con puntos que generan ruido (posiblemente una rama baja o matorral) (c); y sección completa de un árbol sin ruido (d). 


\subsubsection{Identificación de los árboles}

El siguiente paso ha sido la identificación del máximo número posible de árboles detectados por el TLS. Esto se ha obtenido mediante el algoritmo DBSCAN (Ester, et al., 1996) a secciones de la nube de puntos normalizada obtenidas a diferentes alturas $(0.9,1.1,1.3,1.5,1.7 \mathrm{~m} \pm 0.05 \mathrm{~m})$, formando una colección de clústeres que en principio se han considerado como secciones potenciales de árboles (Fig. 3). A continuación, para cada uno de los clústeres se calculó el hipotético centro de la sección, para ello se superpuso una malla cuadrada de $1.5 \mathrm{~cm}$, y para cada punto de intersección de dicha malla se calculó la varianza de sus distancias a todos los puntos del clúster. Sabiendo que el centro es equidistante a todos los puntos de la circunferencia, y suponiendo una sección del árbol circular, se eligió como centro de la sección el punto de intersección de la malla con menor varianza. El radio de la sección fue calculado como la media aritmética de todas las distancias de los puntos del clúster al centro.

Una vez determinados la posición más probable de los centros y los radios de las hipotéticas secciones asociadas a cada clúster, se emplearon una serie de criterios basados en principios geométricos para clasificar los clústeres como árbol o no árbol, para lo que se asumió una disposición circular de los retornos alrededor de la sección normal. Éstos fueron aplicados a modo de árbol de decisión (Fig. 4). En primer lugar, se ha comprobado que el centro de la sección asociada a un clúster no se encontrase entre la posición del TLS y la nube de puntos del clúster. Para ello, se ha comprobado que al menos el $5 \%$ de los puntos más cercanos al TLS tuviesen valores de la coordenada radial $(r)$ menores a la del centro $\left(r_{\text {centro }}\right)$ :

$$
\text { Clúster } \in \text { árbol } \leftrightarrow P_{5}(r)<r_{\text {centro }}
$$

donde $P_{5}$ es el percentil 5 de las coordenadas $r$ de todos los puntos del clúster.

En segundo lugar, y partiendo del principio de que un árbol no debería mostrar puntos de escaneo detrás de la superficie del tronco expuesta al TLS, se ha comprobado que al menos el $95 \%$ de los puntos estuviese a una distancia del centro del clúster superior a la mitad del radio (Fig. 5 b):

$$
\text { Clúster } \in \text { árbol } \leftrightarrow P_{5}(\text { distancias })>\frac{\text { radio }}{2}
$$

donde $P_{95}$ es el percentil 95 de las distancias de los puntos al centro.

El tercer criterio de clasificación se ha basado en que, para los árboles completamente visibles desde el TLS se obtendrían secciones similares a arcos de circunferencia; y por lo tanto las coordenadas $r$ de los extremos de estas secciones deberían estar más alejadas del TLS que las $r$ de la parte central. Con objeto de evitar posibles puntos anómalos, se ha tenido en cuenta el $5 \%$ de los puntos pertenecientes a los extremos y al centro, para así obtener valores promediados (Fig. 5 a):

$$
\text { Clúster } \in \text { árbol } \leftrightarrow \bar{r}_{P_{5}(\theta) \wedge} \quad \bar{r}_{P_{5}(\theta)}<\bar{r}_{P_{47.5-52.5^{(\theta)}}}
$$




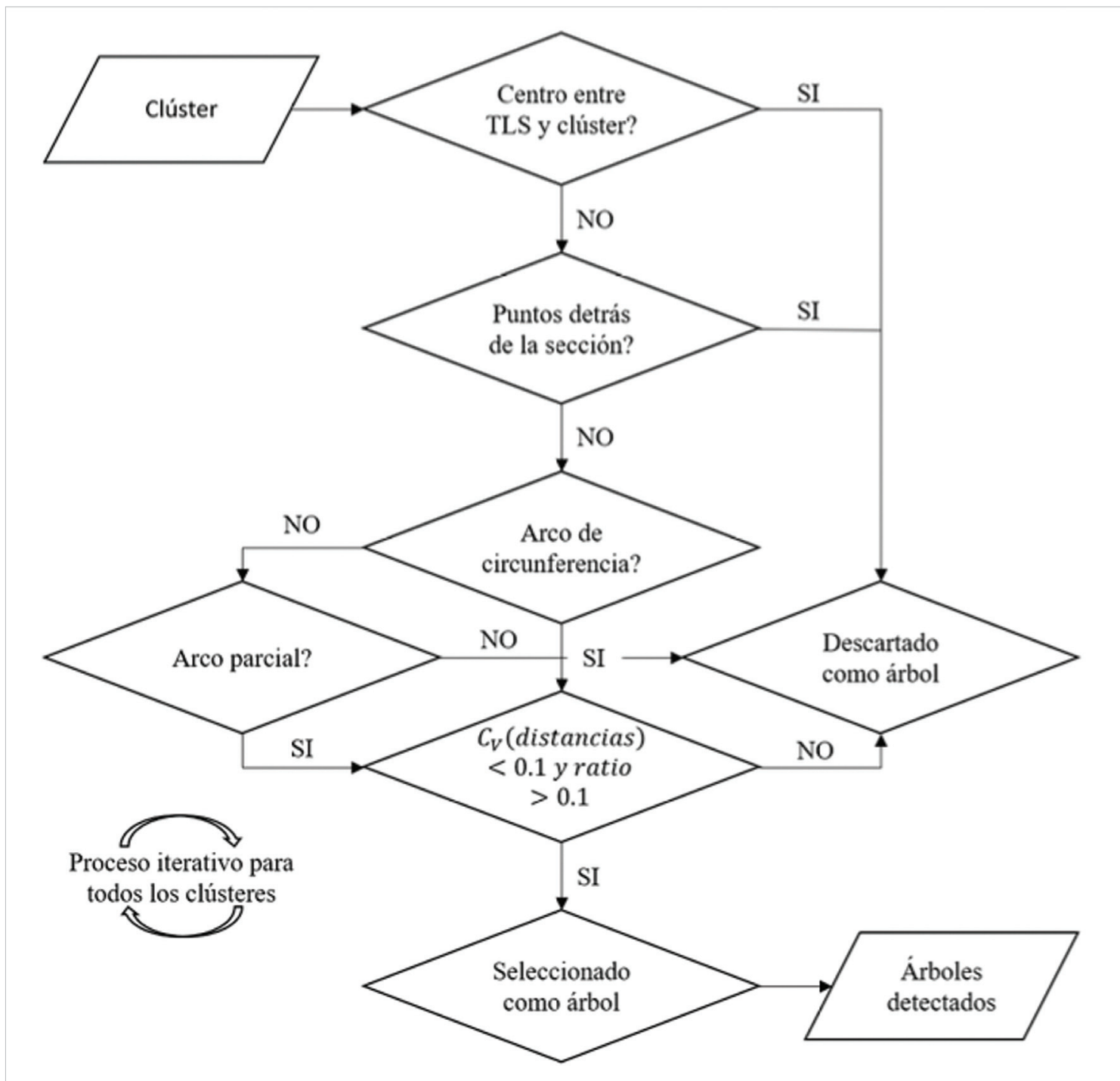

Figura 4. Esquema del algoritmo empleado en cada clúster para la clasificación de estos como árboles.

donde $\bar{r}_{P_{5}(\theta)}$ representa el valor medio de $r$ del 5\% de los puntos del extremo de menor coordenada $\theta, \bar{r}_{P_{95}(\theta)}$ el valor medio de $r$ del $5 \%$ de los puntos de mayor coordenada $\theta$, y $\bar{r}_{P_{47.5-52.5}(\theta)}$ el valor medio de $r$ del $5 \%$ de los puntos centrales.

Para poder comprobar aquellos árboles con oclusiones parciales donde no se ha cumplido el criterio anterior (Fig. 5 c), se ha comprobado que la coordenada azimutal $\theta$ tenga un incremento constante como debería ocurrir para los retornos obtenidos sobre un objeto sólido. Esto se ha evaluado mediante el cálculo de la correlación entre $\theta$ y el ordinal $\left(1,2, \ldots, n_{\text {puntos }}\right)$ de los puntos, seleccionando aquellos clústeres con una fuerte correlación como árboles (Fig. 5 d):

$$
\text { Clúster } \in \text { árbol } \leftrightarrow \rho_{\theta, n}>0.995
$$



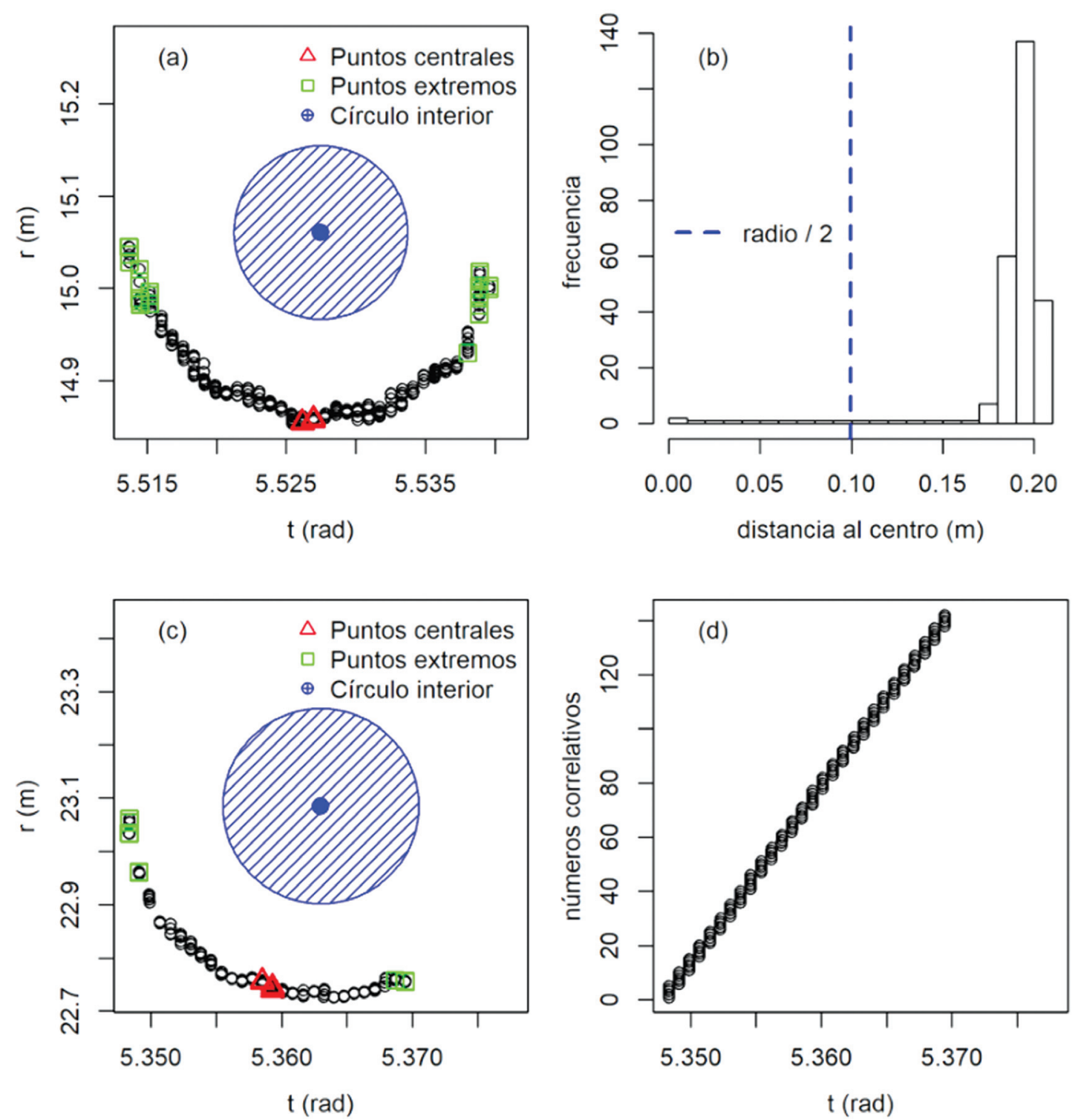

Figura 5. Clústeres seleccionados como árboles y criterios de selección. Árbol sin oclusiones que cumple los criterios de no tener puntos en el círculo interior y ser un arco de circunferencia (a); histograma de frecuencias donde se observa como no hay puntos a la izquierda de la mitad del radio (b); árbol ocluido parcialmente que cumple el criterio de no tener puntos en el círculo interior pero no el del arco de circunferencia (c); árbol ocluido parcialmente que muestra una fuerte correlación positiva entre la coordenada $\theta$ y el ordinal de los puntos. Los puntos extremos (cuadrados verdes) representan los puntos que están por debajo del percentil $5\left(\mathrm{P}_{5}\right)$ y por encima del percentil $95\left(\mathrm{P}_{95}\right)$ en función de la coordenada azimutal $(\theta)$, y los puntos centrales (triángulos rojos) representan los puntos que se encuentran entre los percentiles 47.5 y 52.5 $\left(\mathrm{P}_{47.5}-\mathrm{P}_{52.5}\right)$. El círculo interior representa la sección circular interior del hipotético árbol de radio radio $/ 2$. 
Se ha realizado también un filtrado para descartar aquellos clústeres que mostraron un coeficiente de variación $\left(C_{V}\right)$ de las distancias entre el centro calculado y cada uno de los puntos mayor de 0.1 :

$$
\text { Clúster } \in \text { árbol } \leftrightarrow C_{V}(\text { distancias })<0.1
$$

El siguiente filtrado partió de la hipótesis de que un objeto sólido que no tuviese obstáculos frente al TLS recibirá un número de puntos definido por la resolución del aparato de escaneo, que en este caso es de $7.47 \mathrm{~mm}$ de separación entre dos barridos consecutivos del láser a una distancia de $10 \mathrm{~m}$ del aparato (ecuación 6). Aquellos clústeres con un ratio inferior a 0.1 fueron también eliminados:

$$
\text { ratio }=\frac{\text { numero de puntos del clister }}{\left(\frac{\theta_{\max }-\theta_{\min }}{\alpha}\right)\left(\frac{\varphi_{\max }-\varphi_{\min }}{\alpha}\right)}
$$

donde $\theta_{\max }$ y $\theta_{\min }$ son las coordenadas angulares horizontales máxima y mínima del clúster, $\varphi_{\max }$ y $\varphi_{\min }$ las coordenadas angulares verticales máxima y mínima del clúster, y representa la apertura angular definida para cada barrido del TLS ( $\alpha=$ $\operatorname{arctg}[3.7 / 10,000])$.

$$
\text { Clúster } \in \text { árbol } \leftrightarrow \text { ratio }>0.1
$$

Por último, se eliminaron aquellos posibles árboles para los que $d$ era inferior al $d$ mínimo inventariable $(12.5 \mathrm{~cm})$. En el caso de ser clasificado un clúster como árbol en varias secciones, se ha considerado la sección de $1.3 \mathrm{~m}$ o en su defecto la más próxima.

\subsubsection{Point crooping}

Basado en el principio de que un objeto sólido de un mismo tamaño recibe un mayor número de puntos si se encuentra más cerca del TLS y viceversa, debido a la apertura angular del escaneo; se aplicó un proceso de point crooping para homogeneizar la densidad de puntos en el espacio. El objetivo de este proceso es obtener una nube de puntos en la que el número de puntos sea proporcional al tamaño del objeto, es decir, que la probabilidad de eliminar un punto en la nube original sea inversamente proporcional a la distancia del punto al TLS (Fig. 6). Para ello, se asignó al punto más lejano una probabilidad de selección de $1, \mathrm{y}$ al resto una probabilidad menor definida por el cociente de su distancia radial $(r)$ entre la distancia máxima radial $\left(r_{\max }\right)$ que corresponde al punto más alejado:

$$
\text { Probabilidad de selección }=\frac{r}{r_{\max }}
$$

Para reducir la densidad de la nube de puntos se generó un proceso aleatorio de valores entre 0 y 1 para cada uno de los puntos, de forma que en cada proceso aleatorio fueron seleccionados aquellos con una probabilidad de selección mayor a la obtenida aleatoriamente. 


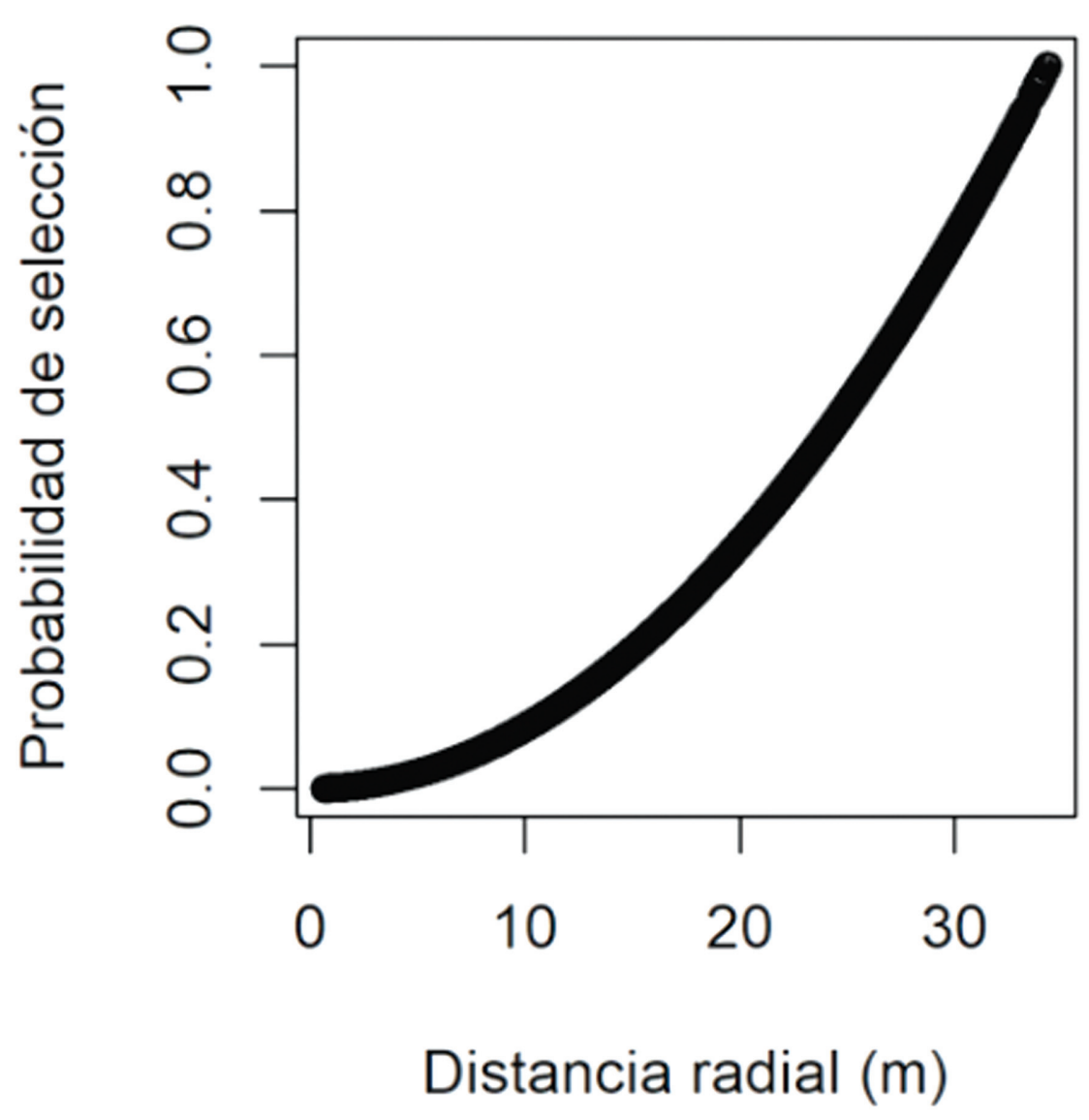

Figura 6. Probabilidad de selección de los puntos en función de la distancia radial.

\subsubsection{Obtención de las métricas}

Con aquellos clústeres que han sido clasificados como árboles se han obtenido las siguientes métricas a nivel de parcela:

1. Cálculo del $G$ con los $d$ estimados (GTLS).

2. Suma del número de puntos pertenecientes a los clústeres con la nube de puntos original (Nиттот).

3. Suma del número de puntos pertenecientes a los clústeres después de aplicar el proceso de point crooping (NUTRED).

\subsection{Análisis de la correlación entre las métricas y los datos de campo}

Para analizar la correlación entre las métricas obtenidas con el TLS (ver sección 2.2.4) y el $G$ estimada en base a las parcelas de campo, primero se ha simulado una 
colección de parcelas circulares de área fija a razón de incrementos radiales de 0.5 $\mathrm{m}$ (radio $=0.5,1, \ldots, 25 \mathrm{~m}$ ). Esto se ha llevado a cabo tanto en las parcelas obtenidas con el TLS como en las de campo, y ha permitido evaluar la variación de la correlación en función del tamaño de parcela considerado. Con las métricas calculadas para cada una de las parcelas simuladas y el valor del $G$ estimada para sus correspondientes de campo, se han analizado 3 escenarios:

1. Estudio de la correlación entre el valor de las métricas obtenidas con el TLS para diferentes tamaños de parcela y el $G$ estimada a partir de la parcela de campo de $10 \mathrm{~m}$ de radio.

2. Estudio de la correlación entre el valor de las métricas obtenidas con el TLS para diferentes tamaños de parcela y el $G$ estimada a partir de la parcela de campo de $25 \mathrm{~m}$ de radio.

3. Estudio de la correlación entre el valor de las métricas obtenidas con el TLS para diferentes tamaños de parcela y el $G$ estimada a partir de sus parcelas correspondientes de campo.

Para estimar el $G$ en función de las métricas obtenidas con el TLS, se realizó un ajuste de regresión lineal simple para el conjunto completo de parcelas utilizando como variable independiente la métrica que mostró una mayor correlación y como variable dependiente el $G$ estimada. También se hizo el ajuste para los valores medios agrupados por sitio, obteniendo un total de 20 sitios de los cuales la mayoría únicamente están representados por una parcela; teniendo en cuenta que el diseño de muestreo no atendió a criterios probabilísticos ni fue diseñado para representar el mayor rango posible de variación de las variables dependientes e independientes. Ello puede haber supuesto que los valores extremos no se encuentren representados en la muestra, y que por lo tanto se vean penalizados los estadísticos de ajuste y capacidad explicativa del modelo. Por último, se calcularon los errores relativos medios cometidos tanto en la colección completa de parcelas como para los valores medios obtenidos por sitio.

\section{Resultados}

El valor de las métricas obtenidas para la estimación del $G$ ha mostrado un comportamiento diferente en todos los escenarios planteados. En el primer escenario, donde se han correlacionado las métricas obtenidas para toda la colección de parcelas TLS con las parcelas campo de $10 \mathrm{~m}$ de radio, se han obtenido las mejores correlaciones cuando existe correspondencia de tamaño, es decir, con la parcela simulada de $10 \mathrm{~m}$ (Fig. 7 a). En concreto ha sido G do una mayor correlación, alcanzando valores significativos para un nivel de confianza del $95 \%$ por encima de 0.8 . En el segundo escenario, donde se han corre- 

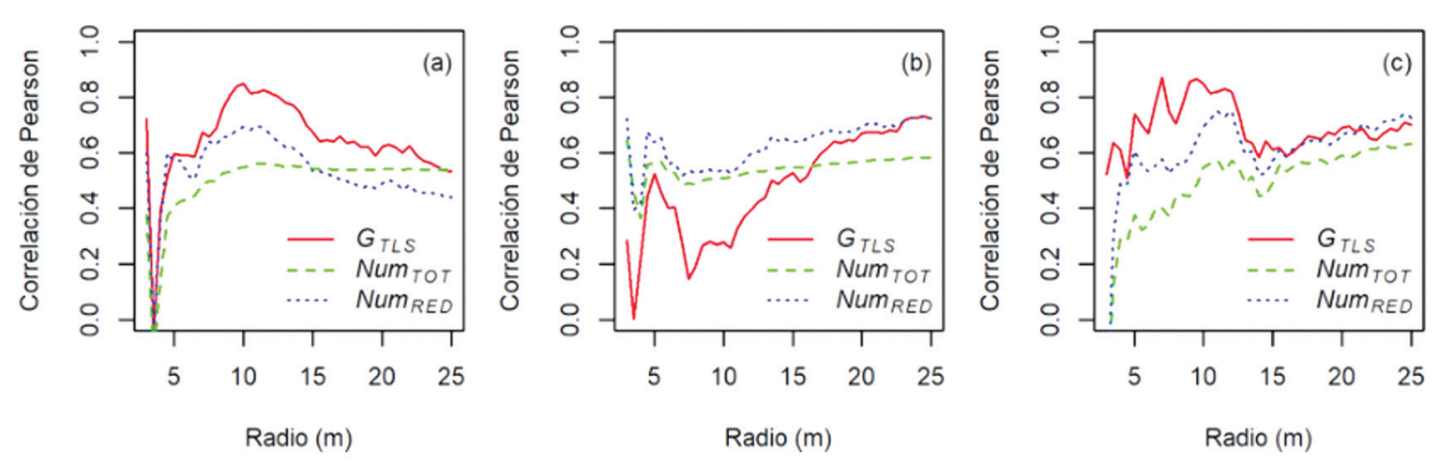

Figura 7. Correlaciones entre el $G$ y las métricas obtenidas con el TLS para: (a) parcela de campo de $10 \mathrm{~m}$ de radio, (b) parcela de campo de $25 \mathrm{~m}$ de radio, y (c) parcelas de campo y TLS correlativas.

lacionado las métricas obtenidas para toda la colección de parcelas TLS simuladas con las parcelas de campo de $25 \mathrm{~m}$ de radio, nuevamente las mejores correlaciones se han dado cuando ha habido correspondencia de tamaño entre parcelas, es decir, con la parcela simulada de $25 \mathrm{~m}$ de radio, pero en este caso tanto GTLS como Num${ }_{R E D}$ han mostrado correlaciones similares (Fig. 7 b). En el último escenario, donde las correlaciones de las parcelas TLS siempre han sido con sus parcelas correlativas de campo, se ha observado una caída importante de la correlación a partir de parcelas de $12 \mathrm{~m}$ de radio en todas las métricas (Fig. 7 c). En este caso ha vuelto a ser $G_{T L S}$ la métrica que mayor correlación ha mostrado y el mejor resultado se ha obtenido para las parcelas de $7 \mathrm{~m}$ de radio con un valor significativo del coeficiente de correlación de Pearson de 0.86. También es destacable que NUm $R E D$ siempre ha mostrado correlaciones mayores que Nиттот.
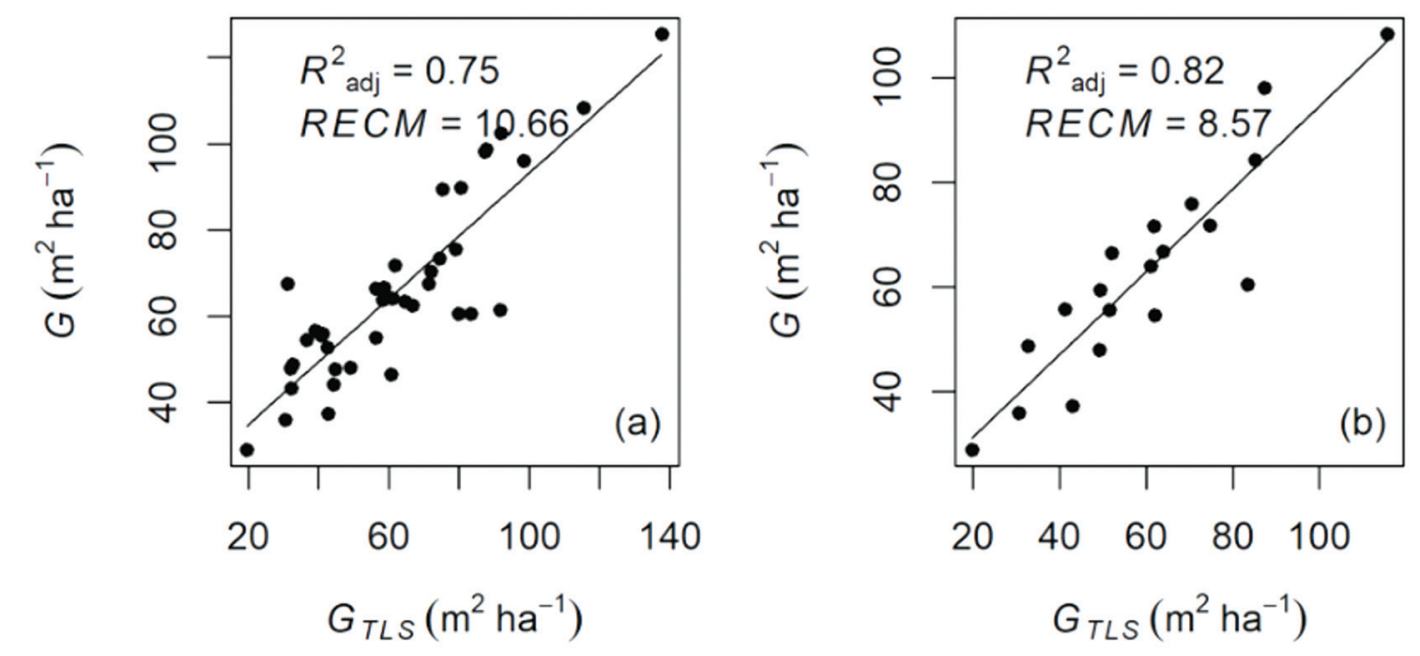

Figura 8. Ajustes de regresión lineal simple entre las parcelas TLS de $7 \mathrm{~m}$ de radio y sus correlativas de campo para: (a) el total de parcelas y (b) los valores medios por sitio. 
A la vista de las correlaciones se ha ajustado un modelo lineal para estimar el valor de $G$ empleando como variable independiente la métrica $G_{T L S}$ de las parcelas de $7 \mathrm{~m}$ de radio. Este mostró un coeficiente de determinación ajustado $\left(R^{2}{ }_{a d j}\right)$ de 0.75 y un valor de la raíz del error cuadrático medio $(R E C M)$ de $10.66 \mathrm{~m}^{2} \mathrm{ha}^{-1}$ (Fig. 8 a). Teniendo en cuenta solamente los valores medios por sitio, también se consiguió un buen ajuste de regresión lineal empleando como variable predictora $G_{T L S}$, con mejores estadísticos que en el caso anterior, con valores de 0.82 para el $R_{\text {adj }}^{2}$ y $8.57 \mathrm{~m}^{2}$ ha $^{-1}$ para el RECM (Fig. 8 b). Los errores relativos medios cometidos en la estimación del $G$ a partir de los modelos ajustados han sido del $12.89 \%$ y $12.86 \%$ para el total de parcelas y los valores medios por sitio, respectivamente (Tabla 3).

Tabla 3. Estadísticos de ajuste de la regresión lineal entre el valor de $G$ y la métrica $G_{T L S}$ y error relativo medio para el total de parcelas y las parcelas de la Umbría de Siete Picos.

\begin{tabular}{lcccc}
\hline Parcelas & $\mathrm{R}^{2} a d j$ & $\mathrm{RECM}\left(\mathrm{m}^{2} \mathrm{ha}^{-1}\right)$ & $\mathrm{p}$-valores & Error relativo medio (\%) \\
Total $(\mathrm{n}=40)$ & 0.754 & 10.660 & $<0.001$ & 12.891 \\
Siete Picos $(\mathrm{n}=7)$ & 0.818 & 8.569 & $<0.001$ & 12.865 \\
\hline
\end{tabular}

\section{Discusión}

A pesar de que las condiciones estructurales de estas masas maduras de $P$. sylvestris han dificultado mucho la detección de los árboles debido principalmente a la presencia de árboles de grandes dimensiones, los cuales causan importantes oclusiones, se han conseguido buenas estimaciones del $G$ con algunas de las métricas para parcelas pequeñas en base a un único escaneo de TLS. En concreto, ha sido la métrica $G_{T L S}$ en parcelas de $7 \mathrm{~m}$ de radio la que mejores correlaciones y ajuste lineal ha mostrado para la estimación del valor de $G$ medido en campo, tanto a nivel de parcela como a nivel de sitio. Estos resultados concuerdan con los obtenidos por Corona et al. (2019), donde fueron las parcelas de $10 \mathrm{~m}$ de radio (las más pequeñas de su estudio) en diferentes tipos de bosques simulados las que lograron la exactitud más alta en la estimación del $G$, tanto con corrección de árboles ocluidos como sin aplicar correcciones. Estos autores sugieren que, en bosques con baja densidad, no está suficientemente justificada la necesidad de aplicar correcciones para los árboles no detectados.

De los resultados obtenidos para los 3 escenarios generados, en primer lugar, se deduce que debe haber una correspondencia entre el tamaño de la parcela de campo y el de la parcela TLS, consiguiendo de este modo una mejora en los resultados en cuanto a la correlación. Por tanto, este debería ser un criterio a tener en cuenta a la hora de diseñar inventarios donde el TLS se use como información complementaria a los datos de campo. Se considera por lo tanto necesario optimizar el tamaño de la parcela mediante un muestreo piloto. En este caso sería aconsejable en la toma de datos en campo anotar la distancia horizontal de los árboles 
al centro de la parcela, de forma que los datos originados con parcelas de grandes dimensiones puedan ser reajustados a parcelas teóricas de menor tamaño, con el objetivo de analizar alternativas para obtener una mayor correlación entre los valores de $G$ medidos en campo y las métricas TLS. Además, la reducción del valor de la correlación de todas las métricas a los $12 \mathrm{~m}$ de radio aproximadamente, precedida por las mayores correlaciones obtenidas, parece indicar la existencia de un posible umbral a partir del cual aumentan las oclusiones. Por último, cabe destacar que la métrica Num RED por lo general se correlaciona mejor con $G$ que la métrica Nuттот. Esto es debido a que de esta forma el número de puntos que aporta cada punto de muestreo es proporcional solamente al tamaño de los árboles, y no a la distancia a la que se encuentran del punto de muestreo. El hecho de que el proceso de point cropping mejore los resultados es una aportación importante de este trabajo y, en cierta medida concuerda con resultados previamente obtenidos en otros estudios con otras variables de interés forestal. Por ejemplo, Torralba et al. (2019) observaron que una reducción progresiva del número de puntos no afectaba en gran medida a las estimaciones de la altura dominante y biomasa, con modelos que explicaban más del $80 \%$ de la variabilidad observada con densidades de hasta 1.5 y 5 puntos $\mathrm{m}^{-2}$ respectivamente. A este respecto hay que tener en cuenta que Torralba et al. (2019) no consideraron en el proceso de reducción de densidad una probabilidad distinta en función de la distancia al TLS, lo que supone que los valores observados por este autor para las estimaciones de masa se vean mejoradas con el procedimiento descrito en el presente trabajo.

El ajuste lineal logrado para el conjunto total de parcelas con un radio de $7 \mathrm{~m}$ ha explicado un $75 \%$ de la variabilidad observada, valor superior al obtenido en Yao et al. (2011) para una colección de 30 parcelas de diferentes tipos de bosques (5 parcelas por sitio y rodal), con formaciones de coníferas y frondosas en diferentes estados de desarrollo y/o gestión. Cuando se ha ajustado la regresión a nivel de sitio los estadísticos $R^{2}$ adj y $R E C M$ han mejorado, alcanzando valores de 0.82 y $8.57 \mathrm{~m}^{2}$ $\mathrm{ha}^{-1}$ respectivamente. Aunque el $R_{a d j}^{2}$ es más bajo que el obtenido en Yao et al. (2011) también a nivel de sitio, hay que tener en cuenta que los diseños de muestreo son diferentes, ya que en Yao et al. (2011) se han implementado muestreos tipo clúster con 5 parcelas circulares de $20-25 \mathrm{~m}$ de radio en cada uno de los sitios.

El error relativo medio para todas las parcelas, con un valor de $12.89 \%$ ha sido menor que el $15.50 \%$ obtenido por Strahler et al. (2008) para una parcela de $50 \mathrm{~m}$ de radio de una plantación tratada de Pinus ponderosa de 30 años. No obstante, hay que tener en cuenta que en Strahler et al. (2008) el G se estimó mediante la simulación de un muestreo relascópico y además se aplicó una corrección de la densidad mediante algoritmos basados en probabilidades geométricas. Sin embargo, Lovell et al. (2011) obtuvieron un error del $4.41 \%$ para la misma parcela, seguramente debido a la mejora del algoritmo. En todo caso, la comparativa entre masas con estructuras y diseños de muestreo tan diferentes no permite extraer conclusiones fundamentadas.

Analizando el error relativo medio a nivel de sitio, su valor se ha mantenido y no ha bajado como cabría esperar a priori y como se ha observado en trabajos pre- 
vios. Por ejemplo, Strahler et al. (2008) en un rodal de 2 ha de Eucalyptus dalrympleana y Eucalyptus delegatensis usado para la producción de madera durante muchos años, reportaron valores de error relativo del $2 \%$ para una media de 8 parcelas relascópicas. No obstante, la mayoría de los sitios muestreados en el presente trabajo son muy heterogéneos debido a una mayor superficie, mayor gradiente altitudinal y mayor variabilidad estructural dentro de cada sitio. Por lo tanto, el muestreo no ha sido diseñado para disminuir el error de muestreo cuando las métricas TLS se usan para asistir la estimación de $G$ mediante la construcción de un modelo, sino que el principal objetivo ha sido muestrear parcelas maduras en máximo stock de carbono registrando el mayor gradiente posible en calidad de estación.

En los últimos años la estimación del valor de $G$ en base a un único escaneo de TLS no se ha estudiado ampliamente en la literatura científica, siendo esta sin embargo una de las principales variables dasométricas que se reportan en un IF. Este trabajo contribuye a mejorar el conocimiento del TLS en la estimación de $G$ en base a un único escaneo, y de esta manera aumentar el interés que despierta su implementación en los IF, el cual pasa por hacer del TLS una herramienta funcional (Liang et al., 2016). No obstante, son necesarios más trabajos similares en diferentes tipos de masas forestales y con diferentes diseños de muestreo, así como considerando la corrección de oclusiones, que sirvan para mejorar la comprensión en la estimación de variables dasométricas con el TLS.

\section{Conclusiones}

Este trabajo muestra que se puede estimar el $G$ en masas maduras de $P$. sylvestris en base a un único escaneo de TLS. Para ello es necesario evaluar el tamaño de parcela más adecuado en base a la correlación entre las métricas obtenidas con el TLS y las estimaciones del $G$, lo cual podría realizarse en base a un muestreo piloto. Se ha demostrado que estas correlaciones adquieren valores máximos cuando las parcelas TLS y las de campo son de las mismas dimensiones, y que para parcelas de más de $12 \mathrm{~m}$ de radio la calidad de las estimaciones baja debido a las oclusiones. Aunque estos resultados son prometedores, es necesario evaluar si estas conclusiones son extensibles a diferentes tipologías de bosque y diseños de muestreo que los considerados en el presente estudio.

\section{Agradecimientos/Financiación}

Juan Alberto Molina-Valero ha sido financiado por el Ministerio de Ciencia, Innovación y Universidades mediante la concesión de una ayuda para la formación del profesorado universitario (FPU 16/03057). César Pérez-Cruzado ha sido financiado por la Comisión Europea a través del programa Marie Sklodowska-Curie (QUAFORD).

Este trabajo se ha desarrollado en el marco de los proyectos nacionales "Mo- 
delización del efecto de la intensidad de perturbación sobre la estructura y el stock de carbono en masas naturales a partir del Inventario Forestal Nacional" (AGL2016-76769-C2-2-R), y del proyecto "Modelización no paramétrica de dinámicas y dependencias en sistemas complejos" (MTM2016-76969-P), ambos concedidos y financiados por la Agencia Española de Investigación.

\section{Bibliografía}

Arias-Rodil, M.; Diéguez-Aranda, U.; Álvarez-González, J.; Pérez-Cruzado, C.; CastedoDorado, F.; González-Ferreiro, E.; 2018. Modeling diameter distributions in radiata pine plantations in Spain with existing countrywide LiDAR data. Ann. For. Sci. 75(2), 36. https://doi.org/10.1007/s13595-018-0712-z

Brede, B.; Lau, A.; Bartholomeus, H.M.; Kooistra, L.; 2017. Comparing RIEGL RiCOPTER UAV LiDAR Derived Canopy Height and DBH with Terrestrial LiDAR. Sens. 17(10), 2371. https://doi.org/10.3390/s17102371

Cabo, C.; Ordóñez, C.; López-Sánchez, C.A.; Armesto, J.; 2018. Automatic dendrometry: Tree detection, tree height and diameter estimation using terrestrial laser scanning. Int. J. Appl. Earth Obs. Geoinformation. 69, 164-174. https://doi.org/10.1016/j.jag.2018. 01.011

Clutter, J.L.; Fortson, J.C.; Piennard, L.V.; Brister, G.H.; Bailey, R.L.; 1983. Timber management: a quantitative approach. John Wiley \& Sons, Inc., New York.

Corona, P.; Di Biase, R.M.; Fattorini, L.; D'Amati, M.; 2019. A Monte Carlo appraisal of tree abundance and stand basal area estimation in forest inventories based on terrestrial laser scanning. Can. J. Forest Res. 49(1), 41-52. https://doi.org/10.1139/cjfr-2017-0462

Ducey, M.J.; Astrup, R.; 2013. Adjusting for nondetection in forest inventories derived from terrestrial laser scanning. Can. J. Remote Sens. 39(5), 410-425

Ester, M.; Kriegel, H.P.; Sander, J.; Xu, X.; 1996. A density-based algorithm for discovering clusters in large spatial databases with noise. Kdd. 96(34), 226-231

Gobakken, T.; Næsset, E.; 2004. Estimation of diameter and basal area distributions in coniferous forest by means of airborne laser scanner data. Scand. J. For. Res. 19(6), 529542. https://doi.org/10.1080/02827580410019454

Guerra-Hernández, J.; Tomé, M.; González-Ferreiro, E.; 2016. Cartografia de variables dasométricas en bosques Mediterráneos mediante análisis de los umbrales de altura e inventario a nivel de masa con datos LiDAR de baja resolución. Rev. Teledetec. 46, 103117. https://doi.org/10.4995/raet.2016.3980

Holmgren, J.; 2004. Prediction of tree height, basal area and stem volume in forest stands using airborne laser scanning. Scand. J. For. Res. 19(6), 543-553. https://doi.org/10. 1080/02827580410019472

Hopkinson, C.; Chasmer, L.; Young-Pow, C.; Treitz, P.; 2004. Assessing forest metrics with a ground-based scanning lidar. Can. J. Remote Sens. 34(3), 573-583. https://doi.org/ $10.1139 / \mathrm{x} 03-225$

Liang, X.; Hyyppä, J.; Kaartinen, H.; Lehtomäki, M.; Pyörälä, J.; Pfeifer, N.; Holopainen, M.; Brolly, G.; Francesco, P.; Hackenberg, J.; Huang, H.; Jo, H.; Katoh, M.; Liu, L.; Mokros, M.; Morel, J.; Olofsson, K.; Poveda-Lopez, J.; Trochta, J.; Wang, D.; Wang, Y.; 
Wang, J.; Xi, Z.; Yang, B.; Zheng, G.; Kankare, V.; Luoma, V.; Yu, X.; Chen, L.; Vastaranta, M.; Saarinen, N.; Wang, Y.; 2018. International benchmarking of terrestrial laser scanning approaches for forest inventories. ISPRS J. Photogramm. Remote Sens. 144, 137-179. https://doi.org/10.1016/j.isprsjprs.2018.06.021

Liang, X.; Kankare, V.; Hyyppä, J.; Wang, Y.; Kukko, A.; Haggrén, H.; Yu, X.; Kaartinen, H.; Jaakkola, A.; Guan, F.; Holopainen, M.; Vastaranta, M.; 2016. Terrestrial laser scanning in forest inventories. ISPRS J. Photogramm. Remote Sens. 115, 63-77. https://doi. org/10.1016/j.isprsjprs.2016.01.006

Lovell, J.L.; Jupp, D.L.B.; Newnham, G.J.; Culvenor, D.S.; 2011. Measuring tree stem diameters using intensity profiles from ground-based scanning lidar from a fixed viewpoint. ISPRS J. Photogramm. Remote Sens. 66(1), 46-55. https://doi.org/10.1016/j.ispr sjprs.2010.08.006

Moskal, L.M.; Zheng, G.; 2012. Retrieving Forest Inventory Variables with Terrestrial Laser Scanning (TLS) in Urban Heterogeneous Forest. Remote Sens. 4(1), 1-20. https://doi. org/10.3390/rs4010001

Newnham, G.J.; Armston, J.D.; Calders, K.; Disney, M.I.; Lovell, J.L.; Schaaf, C.B.; Strahler, A.H.; Danson, F.M.; 2015. Terrestrial Laser Scanning for Plot-Scale Forest Measurement. Curr. Forestry Rep. 1(4), 239-251. https://doi.org/10.1007/s40725-015-0025-5

Olofsson, K.; Holmgren, J.; Olsson, H.; 2014. Tree Stem and Height Measurements using Terrestrial Laser Scanning and the RANSAC Algorithm. Remote Sens. 6(5), 43234344. https://doi.org/10.3390/rs6054323

R Core Team, 2019. R: a language and environment for statistical computing. Vienna, Austria: R Foundation for Statistical Computing.

Roussel, J.; Auty, D.; De Boissieu, F.; Meador, A.; 2019. Airborne LiDAR Data Manipulation and Visualization for ForestryApplications. R package version 2.1.2.

Strahler, A.H.; Jupp, D.L.; Woodcock, C.E.; Schaaf, C.B.; Yao, T.; Zhao, F.; Yang, X.; Lovell, J.; Culvenor, D.; Newnham, G.; Ni-Miester, W.; Boykin-Morris, W.; 2008. Retrieval of forest structural parameters using a ground-based lidar instrument (Echidna ${ }^{\circledR}$ ). Can. $J$. Remote Sens. 34(sup2), S426-S440. https://doi.org/10.5589/m08-046

Torralba, J.; Ruiz, L.A.; Carbonell-Rivera, J.P.; Crespo-Peremarch, P.; 2019. Análisis de posiciones y densidades TLS (Terrestrial Laser Scanning) para optimizar la estimación de parámetros forestales. In: Ruiz, L.A.; Javier Estornell, Abel Calle; Juan Carlos AntuñaSánchez, (eds.), Teledetección: hacia una visión global del cambio climático. 443-446

Van Leeuwen, M.; Nieuwenhuis, M.; 2010. Retrieval of forest structural parameters using LiDAR remote sensing. Eur. J. Forest Res. 129(4), 749-770. https://doi.org/10.1007/ s10342-010-0381-4

Yao, T.; Yang, X.; Zhao, F.; Wang, Z.; Zhang, Q.; Jupp, D.; Lovell, J.; Culvenor, D.; Newnham, G.; Ni-Meister, W.; Schaaf, C.; Woodcock, C.; Wang, J.; Li, X.; Strahler, A.; 2011. Measuring forest structure and biomass in New England forest stands using Echidna ground-based lidar. Remote Sens. Environ. 115(11), 2965-2974. https://doi.org/10. 1016/j.rse.2010.03.019

Zhang, W.; Qi, J.; Wan, P.; Wang, H.; Xie, D.; Wang, X.; Yan, G.; 2016. An Easy-to-Use Airborne LiDAR Data Filtering Method Based on Cloth Simulation. Remote Sens. 8(6), 501. https://doi.org/10.3390/rs8060501 
